\title{
COMMENT NOMMER LES ÉLÉMENTS? LES CATÉGORIES ANTHROPOLOGIQUES EN AFRIQUE DU SUD*
}

\author{
Adam KUPER
}

RÉSumÉ : L'anthropologie sud-africaine a historiquement été divisée en deux écoles : l'une, associée aux universités de langue afrikaans, appuyait l'apartheid; l'autre, associée aux universités anglophones, s'opposait à la ségrégation et à la discrimination raciale. L'anthropologie afrikaner se focalisait sur la culture, la tradition et l'ethnicité, tandis que l'école rivale voulait étudier l'Afrique du Sud comme une société unique, en transformation rapide. Cette opposition, qui a parfois été exagérée, a été la plus marquée dans la période de renforcement de l'apartheid, dans les années soixante et soixante-dix, mais il y a toujours eu un débat considérable sur les objets mêmes des recherches en anthropologie, en particulier sur la nature des groupes « raciaux » et «tribaux » en Afrique du Sud. Ces problèmes, fondamentaux d'un point de vue politique, ont forcé les anthropologues à se confronter aux grandes questions posées par les politiques gouvernementales. Cet article retrace l'histoire de ces débats sur la classification des populations en Afrique du Sud.

Mots-clés : ethnicité, apartheid, histoire de l'anthropologie, Afrique du Sud, Botswana, classification.

ABSTRACT: South African anthropology has been historically divided into two schools. One, associated with the Afrikaans-language universities, was favourable to apartheid. The other, associated with the English-language universities, was opposed to segregation and racial discrimination. Afrikaner anthropology focused on culture, tradition, and ethnicity, while the other school was committed to the study of South Africa as a single, rapidly changing society. This opposition has sometimes been exaggerated, and it was most significant during the period of high apartheid in the sixties and seventies but there has always been considerable debate over the very objects of anthropological research, and in particular about the nature of the « racial» and «tribal " groups in South Africa. These issues were politically of the greatest significance, forcing anthropologists to confront great questions about government policy. This paper traces the history of these debates on the classification of the peoples of Southern Africa.

KEYWORDS : ethnicity, apartheid, history of anthropology, South Africa, Botswana, classification.

* Le titre original du manuscrit de cet article, «Today we have naming of parts », est tiré d'un célèbre poème de Henry Reed sur la Seconde Guerre mondiale, Lessons of the War (1946). Il décrit un instructeur montrant les différentes parties d'un fusil et en indiquant le nom aux recrues.

Revue de synthèse : $4^{\text {e }}$ S. n ${ }^{\text {os }}$ 3-4, juil.-déc. 2000, p. 265-290. 
Zusammenfassung : Die südafrikanische Anthropologie hat sich in ihrer Geschichte in zwei Schulen geteilt. Die eine dieser Schulen befürwortete die Apartheid und war an den Universitäten vertreten, an denen Afrikaans die Unterrichtssprache war; die andere wurde an den englischsprachigen Universitäten gelehrt und lehnte die Rassentrennung und-diskriminierung ab. Die Anthropologie der Afrikaans-Universitäten konzentrierte sich auf Kultur, Tradition und Ethnizität, während die damit rivalisierende Richtung Südafrika als einheitliche, in schneller Umwandlung begriffene Gesellschaft untersuchen wollte. Dieser Gegensatz, der bisweilen übertrieben worden ist, war in der Zeit der Apartheid, d.h. zwischen 1960 und 1970, besonders ausgeprägt, aber es gab immer eine heftige Auseinandersetzung über die eigentlichen Gegenstände der Anthropologie, insbesondere über das Wesen der Rassen - und Stammesgruppen in Südafrika. Diese aus politischer Sicht grundsätzlichen Probleme zwangen die Anthropologen dazu, sich den wichtigen Fragen zu stellen, die mit der Politik der Regierung zusammenhingen. In diesem Artikel werden die Auseinandersetzungen über die Klassifizierung der südafrikanischen Völker dargestellt.

STICHWÖRTER: Ethnizität, Apartheid, Geschichte der Anthropologie, Südafrika, Botswana, Klassifizierung.

RESUMO : Historicamente a antropologia sul-africana esteve dividida em duas escolas : uma, associada às universidades de língua africânder, que era partidária do apartheid, a outra, associada às universidades de língua inglesa, que se opunha à segregação e à discriminação raciais. A antropologia africânder focava sua atenção na cultura, na tradição e na etnicidade, enquanto a outra escola estava voltada para o estudo da África do Sul como uma sociedade singular, em rápida mutação. Esta oposição chegou a ser exacerbada, e foi mais significativa durante o auge do apartheid nas décadas de 60 e 70. Sempre houve no entanto um debate acirrado a respeito dos próprios objetos da pesquisa antropológica, em particular sobre a natureza dos grupos «raciais» $e$ «tribais » na África do Sul. Estes problemas tinham um alto significado político, e os antropólogos se viam confrontados com questões importantes de política de Governo. Este artigo reconstitui a história destes debates a respeito da classificação dos povos na África do Sul.

PalavRas-Chaves : etnicidade, apartheid, história da antropologia, África do Sul, Botswana, classificação.

Adam KuPER, né en 1941 à Johannesburg, a étudié l'anthropologie sociale et l'histoire à l'université de Witwatersrand. Pour son doctorat à l'université de Cambridge, il a réalisé une longue recherche de terrain dans le désert du Kalahari, dans ce qui est aujourd'hui le Botswana. Plus récemment, il a publié des études comparatives d'ethnographie et d'histoire sur des thèmes sud-africains. Il a également beaucoup publié sur l'histoire de l'anthropologie. Il est, depuis 1986, professeur d'anthropologie sociale à l'université de Brunel (Londres).

Adresse: Brunel University, Uxbridge UB8 3PH, G.-B.

Courrier électronique : adam.kuper@brunel.ac.uk 
La transition politique spectaculaire qui a eu lieu en Afrique du Sud au début des années quatre-vingt-dix a suscité, parallèlement à d'autres initiatives plus importantes, une série d'efforts réflexifs parmi les anthropologues. Curieusement peut-être, ceux-ci n'ont pas regardé vers l'avenir, pour voir en quoi l'anthropologie pouvait contribuer à la nouvelle Afrique du Sud. Au contraire, ils ont regardé en arrière en considérant le développement historique de leur discipline dans l'ancienne Afrique du Sud ${ }^{1}$.

Plus précisément, ils ont eu tendance à n'étudier que l'opposition classique entre les deux écoles d'anthropologie sud-africaines. L'une avait été l'alliée de l'apartheid. Son fondateur, Werner Eiselen, premier professeur d'ethnologie dans une université de langue afrikaans (l'université de Stellenbosch), fut l'architecte intellectuel du système de l'apartheid. Étudiant et protégé d'Eiselen, Pieter Coertze développa à l'université de Pretoria un nouveau département influent, et ses étudiants préférés créèrent à leur tour des départements dans d'autres universités de langue afrikaans. Ces ethnologues afrikaners se nommaient eux-mêmes volkekundiges (spécialistes de la science ethnique). Leurs théories concernaient principalement le concept de culture, et leur domaine était la culture et l'ordre social traditionnels des peuples africains d'Afrique du Sud. L'autre école, fondée par Alfred Reginald Radcliffe-Brown, était en réalité une branche locale de l'anthropologie sociale britannique. Associée aux universités de langue anglaise, ses représentants étaient en général opposés à la politique du gouvernement. Ils s'intéressaient à des débats portant sur la structure et la transformation sociales plutôt que sur la culture et la tradition.

L'opposition entre ces deux écoles prit un tour très marqué au cours de la période où l'apartheid était le plus rigoureux, entre les années soixante et quatre-vingt, à une époque où chacune était identifiée à une position politique antithétique à l'autre, pour ou contre le gouvernement ${ }^{2}$. Une anthropologue africaine de renom, Mamphela Ramphele, se souvient que lorsque Thabo Mbeki, l'actuel président de l'Afrique du Sud, revint d'exil, il lui reprocha de vouloir devenir anthropologue. À ses yeux, la discipline

1. Pour plus de précisions sur les références citées en notes, se reporter à la liste finale, p. 289-290. Ici, Gordon, 1990; Gordon et Spiegel, 1993; Hammond-Tooke, 1997; Kiernan, 1997; Kuper, 1999, chap. ix; Ramphele, 1995; Van Rensburg et Van der WaAl, 1999; VAWDA, 1995.

2. C'est WEST, 1988, qui a le plus clairement montré cette opposition entre l'anthropologie anglaise et l'anthropologie afrikaner en Afrique du Sud lors de sa leçon inaugurale comme professeur d'anthropologie sociale à l'université de Cape Town. 
avait été irrémédiablement compromise par son passé colonial. Elle se souvient avoir « répondu avec assurance qu'il lui fallait distinguer entre la bonne et la mauvaise anthropologie ».

«Bien qu'une forme particulièrement maligne d'anthropologie en vigueur dans certaines universités de langue afrikaans ait fourni une justification ethnologique à la ségrégation, il existe une autre tradition qui a valu à l'anthropologie sud-africaine d'être honorée sur le plan international. Radcliffe-Brown, Monica Wilson, les Mayers et bien d'autres ont accompli un travail de qualité qui a permis une compréhension à la fois meilleure et plus subtile de la société sud-africaine ${ }^{3}$. »

Toutefois, au cours des années quatre-vingt-dix, cette opposition simple entre une bonne anthropologie anglophone et une mauvaise anthropologie afrikaner commença à apparaître quelque peu caricaturale. D'abord, elle faisait abstraction de ce que certains des meilleurs chercheurs des années trente n'appartenaient à aucun des deux camps de façon claire : Nicholas J. Van Warmelo par exemple, ethnologue officiel du gouvernement, avait de nombreux points communs avec Isaac Schapera, l'héritier de RadcliffeBrown, ce que les deux hommes reconnaissaient volontiers. Après la guerre, les divisions s'intensifièrent, mais le doyen des anthropologues anglophones d'Afrique du Sud de l'après-guerre, W. David HammondTooke, commença ses études universitaires sous la direction de Schapera; il rédigea ensuite une thèse sous la direction de Wilson, pour faire enfin sa période d'apprentissage dans la section ethnologique du département des affaires indigènes sous la direction de Van Warmelo, qu'il respectait ${ }^{4}$. De plus, s'il est vrai que l'Association de langue anglaise pour l'anthropologie sociale (Association for Social Anthropology) dans le sud de l'Afrique accueillait des Noirs parmi ses membres, et que des chercheurs noirs furent nommés à des postes haut placés aux universités de Cape Town et de Witwatersrand dans les années quatre-vingt, les anthropologues non blancs des Bush colleges se sentaient marginalisés et se plaignaient qu'on ne tenait pas compte de leurs préoccupations ni de leurs contributions ${ }^{5}$.

Il est aussi trop simple, et peut-être très commode, de diaboliser les spécialistes de la science ethnique afrikaners. Les institutions nationalistes imposaient en ethnologie une orthodoxie rigoureuse. En effet, tous les professeurs d'ethnologie des universités de langue afrikaans étaient membres d'une société secrète, la Fraternité (Broederbond), qui fonctionnait comme l'institution interne du mouvement nationaliste. La critique de l'apartheid

3. Ramphele, 1995, p. 164-167.

4. HAMmond-ToOKe, 1997, chap. v.

5. Voir, p. ex., VAwda, 1995. 
n'était pas tolérée, et le rapprochement avec les anthropologues anglophones fermement déconseillé. D'autre part, certains spécialistes de la science ethnique manifestèrent une sympathie limitée envers des théories américaines traditionnelles en anthropologie culturelle, et même avec les idées théoriques de Bronislaw Malinowski ${ }^{6}$. Tous n'étaient pas des racistes fanatiques, encore moins des crypto-nazis (encore qu'ils accueillirent quelques ethnologues allemands au passé douteux qui arrivèrent en Afrique du Sud peu après 1945).

Cette opposition entre les deux traditions anthropologiques en Afrique du Sud avait néanmoins une part indubitable de réalité, particulièrement du point de vue des praticiens dans les universités sud-africaines des années soixante-dix et quatre-vingt. Toutefois, insister sur ces différences, qui ont bien existé, pourrait cacher le fait que les anthropologues sud-africains de toutes convictions politiques participaient à un dialogue les uns avec les autres et, plus important encore, avec ceux qui décidaient de la politique gouvernementale. Peut-être le terme de dialogue est-il trop faible pour ce qui était une confrontation politique âpre, pleine de problèmes et parfois dangereuse, mais ce qui est à souligner, c'est que le discours de l'anthropologie sud-africaine était nécessairement marqué par les luttes politiques. Il en allait inévitablement de la sorte pour les serviteurs de l'apartheid, mais cela concernait aussi les adversaires les plus acharnés de ce dernier. Peu d'anthropologues purent éviter de prendre position dans le champ de bataille en s'exprimant plutôt en référence aux débats universitaires internationaux. Hammond-Tooke, par exemple, tenta de frayer un chemin vers des domaines nouveaux de l'anthropologie sud-africaine moins sensibles politiquement, mais à mesure que la confrontation politique devenait de plus en plus aiguë, ses quelques alliés et lui-même se retrouvèrent isolés et ignorés ${ }^{7}$.

Le grand débat portait sur la race et la culture, la tradition et le changement mais, de manière plus fondamentale, il concernait le caractère même de la société sud-africaine et la nature de ses éléments. À n'en pas douter, la question la plus fondamentale opposant les anthropologues dans toute société a toujours été la définition des unités de base de la vie humaine. Faut-il grouper les gens selon des critères de race, de descendance, de langue, de coutumes, de religion, d'orientation politique? Quels sont les groupes sociaux de base, la tribu, le clan, la caste, le groupe ethnique ou la

6. VAN Rensburg et VAn der WAal, 1999.

7. Hammond-ToOKe, 1997, chap. viII; Kiernan, 1997. 
nation? Pour compliquer les choses davantage, il peut y avoir des affiliations multiples. De plus, ces unités peuvent représenter elles-mêmes les éléments de systèmes sociaux plus complexes. À l'inverse, elles peuvent n'être que les subdivisions internes d'un ordre économique ou politique plus vaste, plus global, qui les situe et les modèle selon ses intentions. Dans ce cas, il devient primordial de déterminer si ces structures sont mieux comprises en tant qu'États coloniaux ou capitalistes, en tant que sociétés plurielles, multiculturelles ou multiraciales, ou encore en tant que nations embryonnaires.

Ce sont là des questions universelles, qui ont toujours défini les débats anthropologiques. Néanmoins, il y a peu de régions du monde dans lesquelles la caractérisation des identités ethniques a été plus chargée de problèmes et a le plus tragiquement contribué à la formation d'une nation qu'en Afrique du Sud. Ces identités ont été aussi remarquablement instables. Aujourd'hui, il est tentant de croire que l'histoire sud-africaine a toujours été modelée par une théorie des races qui a opposé les Blancs aux Noirs, mais en réalité les divisions de l'Afrique du Sud ont toujours été formées et comprises de façon variée, et âprement contestées.

Sous la domination de la Compagnie hollandaise des Indes orientales (1652-1795), les oppositions de base se situaient entre chrétiens et paiens, entre esclaves et hommes libres. Pendant la période hollandaise, les théories racistes n'étaient ni très influentes ni même clairement formulées, et le statut social ne dépendait pas de la couleur de la peau ou de l'origine : les Chrétiens libres (Free Christians) étaient acceptés à tous les niveaux de la société. Selon Robert Ross, «le système esclavagiste a constitué paradoxalement un frein au développement d'un ordre racial $^{8} »$.

Au début du XIX ${ }^{\mathrm{e}}$ siècle, Le Cap devint une colonie britannique, et l'ordre ancien fut renversé. Les dirigeants britanniques n'avaient guère de sympathie pour les fermiers néerlandophones, les Boers, et leurs décisions politiques adoucirent quelque peu la vie des esclaves et des indigènes du Cap occidental. En 1828, les hommes de couleur libres, notamment les Hottentots, furent affranchis de toutes les incapacités légales. Événement d'une plus grande portée encore, tous les esclaves de l'Empire britannique furent libérés le $1^{\text {er }}$ décembre 1834 . Ces initiatives étaient dues pour une large part aux pressions exercées par les sociétés de missionnaires, qui étaient actives depuis le début du XIX ${ }^{\mathrm{e}}$ siècle. À la même époque, les missionnaires sur le terrain commencèrent aussi à entraîner des conversions de masse des indigènes, ce qui contribua à éroder l'ancienne dichotomie entre les chrétiens et les païens.

8. Ross, 1993, p. 5. Je suis ici de très près l'analyse de Robert Ross, qui est développée notamment dans le chapitre III de son recueil d'essais. 
CATÉGORIES DE POPULATIONS EN AFRIQUE DU SUD

TABLEAU RÉCAPITULATIF

PÉRIODE COLONIALE HOLLANDAISE (MILIEU XVII ${ }^{\mathrm{e}}$-DÉBUT XIX ${ }^{\mathrm{e}}$ SIÈCLE)

\section{Free Christians}

Dutch

Boers (fermiers blancs)

Free Coloured

Autres

Esclaves

Hottentots/Bushmen

(Le terme Kaffir était parfois utilisé de façon interchangeable avec Hottentot mais pouvait aussi être utilisé pour désigner les populations noires au-delà de la colonie.)

PÉRIODE COLONIALE BRITANNIQUE (XIX ${ }^{\mathrm{e}}$ SIÈCLE)

\section{Europeans}

British

Boers

Autres

Native (divisés en Kaffir et Bechuana)

Coloured (au Cap occidental)

Hottentots (fondus de plus en plus avec la catégorie de Coloured)

Bushmen (à la périphérie de la colonie)

Indians (au Natal)

$\mathrm{XX}^{\mathrm{e}}$ SIÈCLE

European/White

Afrikaner

English

Non-European/Non-White

Native, Bantu ou African

Coloured

Asian 
Les Boers étaient hostiles à ces améliorations du statut légal des esclaves et des Hottentots. Du point de vue des fermiers boers de l'intérieur des terres, il existait une opposition évidente, d'origine divine, entre maître et serviteur. Celle-ci se manifestait par des différences dans la couleur de peau, la langue, la manière de vivre et la religion. C'était là une vision typiquement boer, inspirée de la doctrine calviniste de l'élection et de la prédestination. Toutefois, après l'installation en 1820 de colons anglais sur la frontière orientale de la colonie, l'administration britannique fut soumise à une plus forte pression pour soutenir les intérêts des colons blancs. Une justification idéologique séculière pour ce changement de politique fut alors trouvée sous la forme des nouvelles théories scientifiques des races qui se répandaient en Europe. Vers 1850, la race constituait une base de stratification aux yeux des colons blancs comme à ceux des puissances coloniales.

Dans le temps même où la distinction raciale fondamentale entre Blancs et Noirs s'imposait, elle dut être modifiée pour tenir compte des subdivisions qui s'établissaient selon des critères différents (voir tableau récapitulatif, p. 271). La catégorie blanche se divisait en deux nations ou races, les Boers et les Britanniques (Britons), division qui s'établit aux cours du premier tiers $\mathrm{du} \mathrm{XIX}^{\mathrm{e}}$ siècle, à mesure que les agriculteurs d'origine hollandaise aux limites des zones conquises contestaient la domination britannique. Au milieu du $\mathrm{XIX}^{\mathrm{e}}$ siècle, des républiques boers indépendantes furent créées à l'intérieur du pays et une nouvelle colonie britannique, le Natal, fut établie au sud de l'État zoulou. Après la découverte d'or dans les républiques boers, le pouvoir impérial contesta leur existence. Au tournant du siècle, une guerre décisive eut lieu entre l'Empire britannique et les républiques.

La guerre se termina avec la défaite des Boers et l'établissement d'un nouvel État en 1910, l'Union sud-africaine. Le droit de vote en général et aux élections de l'administration communale de l'Union fut effectivement restreint aux Blancs, et la politique électorale dans le nouvel État en vint à être dominée par une opposition entre les «Anglais » et un groupe qui commençait à se donner le nom d'Afrikaners (Africains). Petit à petit, les anciens Boers se considérèrent comme une nation sinon indigène, du moins locale au sein de l'État sud-africain, une image qu'ils s'attribuèrent euxmêmes. Cette image fut ensuite en quelque sorte leur symbole et fut renforcée quand, en 1920, l'afrikaans, la variante locale du hollandais, remplaça le hollandais lui-même comme langue officielle ${ }^{9}$. Au sein de l'Union, le nationalisme afrikaner devint une force politique importante; un parti nationaliste afrikaner parvint au pouvoir dans une coalition gouverne-

9. Le dictionnaire officiel afrikaans, le Woordeboek van die Afrikaanse Taal (Pretoria, 1950), définissait l'Afrikaner de deux façons, en tant que catégorie raciale ou culturelle. «Individu qui est Afrikaans par descendance ou par naissance; individu qui appartient au groupe de la population qui parle l'Afrikaans. » 
mentale entre 1924 et 1939 , et se maintint au pouvoir à nouveau comme parti unique de gouvernement de 1948 à 1994.

De l'autre côté de la division raciale, la situation était encore plus complexe. La colonie du Cap, le Natal et les républiques boers avaient développé des traditions administratives divergentes, et leurs politiques envers les indigènes différaient notablement. Il y eut des débats au Natal et au Cap oriental pour savoir si on devait attribuer aux indigènes «chrétiens » ou «civilisés » un statut spécial, tandis que les anciennes républiques boers, le Transvaal et l'État libre d'Orange insistaient pour avoir une ségrégation stricte ${ }^{10}$. Au Cap occidental, les indigènes étaient une minorité, et les Coloureds (gens de couleur) représentaient le plus grand groupe de population. Tandis que seule une poignée de Coloureds avait obtenu le droit de vote, il n'y avait que peu de restrictions légales concernant la résidence et le travail, état de choses qui était considéré comme scandaleux par les habitants du Transvaal. Au Natal, les hindous pauvres, ouvriers agricoles des plantations, étaient soumis à des entraves légales et sociales, entraves qui ne touchaient pas les communautés plus réduites en taille mais plus riches des commerçants musulmans du Transvaal, tandis qu'il n'était même pas permis aux « Indiens » de passer la nuit sur le territoire de l'État libre d'Orange.

Il n'y avait pas que les pratiques politiques qui étaient différentes. Ces pratiques politiques elles-mêmes dépendaient des classifications raciales et culturelles, et les renforçaient, et ces classifications variaient aussi d'une région à l'autre. La formation de l'Union imposa alors la nécessité d'un système national de catégories raciales comme condition préalable au développement d'une politique administrative uniforme ${ }^{11}$. La solution officielle fut de diviser les «non-Blancs » en trois catégories : les Coloureds, les Asiatiques, et finalement les indigènes, connus aussi sous le nom de Bantous ou Africains. Ces catégories étaient ordonnées selon une hiérarchie grossière où les indigènes occupaient la position la plus basse. Cependant, chacune de ces catégories était en elle-même extrêmement problématique.

Initialement, les termes Hottentot et Kaffir étaient utilisés sans distinction pour désigner la population indigène, mais au milieu du XVIII ${ }^{\mathrm{e}}$ siècle la convention s'établit de diviser les populations indigènes d'Afrique du Sud en deux groupes, Hottentots et Kaffirs. Le terme Hottentot était appliqué aux peuples indigènes «jaunes » du Cap occidental, site de la colonie ori-

10. Pour une discussion en détail des différentes traditions de l'administration indigène avant l'Union, voir HAMiLton, 1998, chap. III et IV.

11. Il serait très intéressant de faire une étude des changements de classification raciale dans les recensements d'Afrique du Sud et de la colonie du Cap. Dans le dernier recensement de la colonie du Cap, en 1904, il y avait sept catégories principales divisées en quarante subdivisions. 
ginelle. Cette population était constituée de petits groupes de pasteurs, de chasseurs et de ramasseurs de coquillage (strandlopers). Sur la frontière orientale de la Colonie, on trouvait des populations «noires» qui différaient des Hottentots par leur apparence, ainsi que par la langue, l'organisation économique et politique. Les pasteurs et les agriculteurs possédaient une technologie élaborée du fer, et étaient organisés en chefferies souvent redoutables. On les appelait Kaffirs, terme utilisé par les Portugais et les Hollandais pour désigner les peuples avec lesquels ils étaient entrés en contact en Afrique orientale avant que la côte sud-africaine fût explorée ${ }^{12}$.

Chacune de ces grandes catégories fut ensuite divisée à nouveau en deux sections. Au XVIII ${ }^{e}$ siècle, les peuples du Cap occidental furent subdivisés en Boschimans (Bushmen) et en Hottentots. Conformément à la pensée des Lumières, le critère pour distinguer ces populations était un critère économique. Les Boschimans étaient des chasseurs, les Hottentots étaient un peuple de pasteurs. Le terme de Kaffir en vint progressivement à ne s'appliquer qu'aux chefferies sur le littoral oriental. D'autres chefferies de même nature dans le Highveld (haut plateau), touchées par la culture européenne au $\mathrm{XIX}^{\mathrm{e}}$ siècle, furent désignées sous le terme de Bechuanas. Une autre source conceptuelle justifia cette distinction entre Kaffirs et Bechuanas : la philologie, science prestigieuse dans la première moitié du XIX ${ }^{e}$ siècle. Les critères linguistiques se répandirent parallèlement aux critères économiques établis pour la classification des peuples. On distingua les Kaffirs des Bechuanas parce que leurs langues appartenaient à deux familles (en réalité très proches), et on interpréta ce phénomène comme l'indice d'une origine séparée.

Cette quadruple classification survécut dans le monde savant au $\mathrm{xx}^{\mathrm{e}}$ siècle, mais avec l'addition de nouvelles catégories plus élevées qui restauraient la division binaire originelle des indigènes. En 1928, un anthropologue physique allemand, Leonard Schultze, introduisit le terme de Khoisan pour décrire ce qu'il prenait pour la souche raciale commune des Boschimans et des Hottentots; en 1930, Schapera reprit ce néologisme dans sa synthèse ethnologique qu'il intitula The Khoisan Peoples of South Africa. Bushmen and Hottentots. Cependant, au début du $\mathrm{xx}^{\mathrm{e}}$ siècle, les Hottentots et encore davantage les Boschimans étaient considérés comme des peuples primitifs éloignés et insignifiants qui vivaient en marge de la société. Les Boschimans avaient été traités en ennemis de la société des colons au cours du $\mathrm{XIX}^{\mathrm{e}}$ siècle, et ils furent parfois pourchassés jusqu'à extinction presque totale. Au $\mathrm{xx}^{\mathrm{e}}$ siècle, on les évoquait pour parler d'un peuple primitif en voie de disparition, pittoresque sans doute, mais d'un

12. Kafir, ou incroyant, était le terme utilisé par les Arabes pour les peuples de l'intérieur de l'Afrique; ce terme fut adopté par les Portugais et les Hollandais. 
intérêt principalement scientifique. Les peuples qu'on désignait autrefois du terme de Hottentots parlaient en général afrikaans et étaient chrétiens, et ils furent progressivement assimilés aussi bien dans le vocabulaire quotidien que dans le discours officiel à la catégorie des Coloureds du Cap occidental, à l'instar de la population des anciens esclaves ${ }^{13}$. Le terme Hottentot était désormais devenu un terme d'injure.

La désignation de Coloured - ou so-called Coloured, dans la terminologie des contestataires - devint courante au milieu du XIX ${ }^{\mathrm{e}}$ siècle. À la suite de l'abolition de l'esclavage et du passage à la législation destinée à en finir avec la discrimination envers les Hottentots, une description ouvertement raciale fut employée pour désigner les habitants du Cap occidental qui n'étaient ni boers ni britanniques, et qu'on avait auparavant distingués sous les catégories esclave, Hottentot, ou encore Baster ${ }^{14}$. Au xx ${ }^{\mathrm{e}}$ siècle, les gens désignés comme Coloureds parlaient principalement l'afrikaans; ils étaient en général membres de congrégations (ségrégées) de l'église réformée de Hollande. Ils portaient des noms afrikaans, et avaient développé un sentiment d'identité en tant qu'Afrikaners bruns, renforcé par la croyance que leurs origines remontaient à des ancêtres blancs afrikaners, ce qui posait un problème à la théorie selon laquelle race et culture coïncident.

$\mathrm{Au}$ Natal et dans le Transvaal, il y avait en outre une population asiatique ou indienne (ou, selon l'usage familier et péjoratif pour les désigner, des coolies), et celle-ci était au moins aussi hétérogène. Cette appellation recouvrait les descendants hindous de paysans du sud de l'Inde engagés dans les plantations du Natal, des Chrétiens libres instruits, et des commerçants musulmans parlant le pendjab, descendants d'immigrants indépendants, qui travaillaient pour l'essentiel dans de petits négoces, et qui s'étaient établis principalement dans le Transvaal. Mahatma Gandhi, qui arriva en Afrique du Sud en 1893 pour y travailler comme avocat, fonda le Congrès indien du Natal en 1894, et resta deux décennies dans le pays comme dirigeant du mouvement politique indien d'Afrique du Sud. Toutefois, jamais une identité politique asiatique ou indienne ne s'est véritablement développée en Afrique du Sud.

13. Les esclaves eux-mêmes n'étaient pas une catégorie ethnique homogène. Bien que la plupart des esclaves aient été amenés des Indes orientales hollandaises, une grande partie provenait de Madagascar et de l'île Maurice. Une nouvelle catégorie apparut au Cap, définie par la religion. Il s'agissait des musulmans du Cape Malays. Ils vivaient en plein centre de Cape Town et descendaient des artisans esclaves qui avaient été importés des Indes orientales hollandaises.

14. Le terme Baster (bâtard), fréquent au XIX ${ }^{\mathrm{e}}$ siècle, s'appliquait aux groupes indépendants d'origine khoï mais de culture boer qui vivaient au-delà des frontières de la Colonie. On l'utilisait aussi pour désigner les gens d'origine mixte (les esclaves et Hottentots, ou les descendants de couples mixtes Blanc-esclave ou Blanc-Hottentot). 
Les Asiatiques et les Coloureds posaient problème pour les classifications administratives ou scientifiques. Du point de vue de l'État et de l'électorat blanc toutefois, les catégories importantes étaient d'une part les Blancs, définis en simples termes de race, et d'autre part les indigènes, catégorie qui comprenait les Kaffirs et les Bechuanas d'autrefois. Ces indigènes représentaient approximativement les trois quarts de la population totale d'Afrique du Sud. Vers la fin du XIX ${ }^{\mathrm{e}}$ siècle, on estimait qu'ils appartenaient à une seule et même race, ainsi qu'à une vaste tradition linguistique et culturelle unique, qui s'étendait très au nord de l'Afrique du Sud. Les linguistes ont alors donné à la grande famille de langue africaine, à laquelle les groupes d'Afrique du Sud appartenaient, le nom de Bantous, terme qui comprenait les peuples sud-africains et la population africaine au sud d'une ligne allant du sud du Cameroun à l'ouest au sud du Kenya à l'est. Dans les années vingt, les universitaires sud-africains se mirent à favoriser Bantou comme le terme générique désignant les anciens indigènes d'Afrique du Sud ${ }^{15}$. Dans les années soixante, cet usage fut adopté par le gouvernement nationaliste.

Mais si, d'un certain point de vue, les Bantous d'Afrique du Sud étaient perçus comme formant un seul groupe, on estimait néanmoins qu'ils étaient également divisés en différentes tribus, à l'exception d'une catégorie problématique d'indigènes détribalisés. Ces tribus étaient considérées comme les divisions sociales et nationales fondamentales des Bantous, mais la définition et la portée de ces unités tribales devinrent extrêmement débattues. Il existait deux modes concurrents pour classer la population indigène ou bantoue. L'un se fondait sur des critères linguistiques, auxquels s'ajoutaient généralement des observations sur la culture et la race. L'autre approche se fondait sur la croyance selon laquelle la condition naturelle des Africains était de vivre sous l'autorité de chefs; en conséquence, c'était leur affiliation tribale qui primait. Toutefois, les tribus ou chefferies, comme on appelait ces unités politiques, ne coïncidaient pas exactement avec les divisions culturelles ou linguistiques. Des communautés qui étaient, de façon évidente, d'origines différentes, se distinguant par leur langue et leur culture, se retrouvaient souvent sous le contrôle d'un chef unique. La communauté naturelle indigène, pour diverses que soient ses origines, se composait-elle des sujets d'un chef, ou bien représentait-elle un groupe de descendance, même si elle ne pouvait pas constituer une communauté politique? Existait-il des nations ou des groupes culturels qui incluaient un certain nombre de chefferies?

15. En 1921, fut fondée en Afrique du Sud une revue universitaire consacrée à l'étude des peuples indigènes du pays. Elle fut nommée Bantu Studies (Études bantoues), mais son but était de promouvoir «l'étude scientifique des Bantous, des Hottentots et des Boschimans ». 
Le missionnaire anthropologue Henri Junod étudia la tribu thonga, qui vivait de part et d'autre de la frontière entre le Mozambique et le Transvaal, d'une population qu'il estimait à 750000 individus. Sur des bases linguistiques et culturelles, ces gens furent divisés en six groupes principaux et en un grand nombre de chefferies ${ }^{16}$. Il admettait que le terme collectif Thonga n'était pas utilisé par le peuple lui-même, mais lui était appliqué par ses voisins; en réalité, comme il apparut par la suite, il s'agissait d'un terme péjoratif Shona pour désigner les gens qui n'ont pas de bétail. Junod reconnut aussi que les différentes parties de la tribu thonga n'avait pas de tradition d'identité commune, qu'il n'y avait pas de nom indigène pour désigner le groupe dans son ensemble, et que les divers clans, ou divisions politiques, faisaient dériver leur origine de traditions différentes. De plus, il estimait que tous les Thongas parlaient des dialectes issus d'une même langue, et avaient en commun des traits culturels qui les distinguaient de ceux qui parlaient zoulou plus au sud, avec lesquels ils étaient par ailleurs indéniablement liés. La logique interne de cette classification n'est pas éblouissante. Toutefois, on a pu dire que sa pertinence tenait à des considérations plus pratiques : la classification de Junod correspondait au domaine d'opérations de sa société missionnaire, la Mission suisse romande. Les missionnaires ont standardisé la langue, et encouragé les sentiments d'unité linguistique et culturelle. Volontairement ou non, ils étaient en train de participer à la création d'une nation ${ }^{17}$.

La tentative la plus sophistiquée pour classer les tribus bantoues d'Afrique du Sud fut entreprise par Van Warmelo, dans une étude publiée en 1935, qui démontrait la difficulté de cette entreprise. Ayant obtenu son doctorat à l'Institut colonial de Hambourg, il avait avant tout une formation de linguiste; mais il employa des critères historiques et ethnologiques, s'intéressa à l'utilisation des sols et au contexte politique de l'époque, et attira l'attention sur ce qu'il nomma « la décadence tribale ${ }^{18} »$. En discutant ses critères de classification, Van Warmelo suggérait qu'une approche purement historique n'était pas possible : les sources étaient inadéquates et, de toute façon, les tribus s'étaient scindées, avaient migré, absorbé des éléments étrangers, avaient été conquises et dispersées par la force, et leur culture même avait changé. Une classification selon des traits culturels n'était pas possible en pratique, puisque notre savoir comportait trop de lacunes et que beaucoup de choses avaient changé dans de nombreux domaines. Il restait la langue, base la plus fiable pour une classification. «Car dans le même temps où de nombreuses tribus avaient été dispersées, et où leur culture avec tous ses signes extérieurs visibles, tels que les vil-

16. JUNOD, 1912, vol. 1, p. 13-19.

17. HARRIES, 1989 , p. 85-90.

18. Van Warmelo, 1935, p. 5. 
lages et les produits artisanaux, avait été détruite, la langue survécut avec ceux qui la parlaient $[\ldots]^{19}$.»

Bien qu'une logique simple de discrimination raciale ait fourni le cadre de base de la classification de la population d'Afrique du Sud au $\mathrm{xx}^{\mathrm{e}}$ siècle, il est clair que les principes qui sous-tendaient cette classification étaient variés et même contradictoires. Toutefois, et en dépit du fait que ces distinctions catégorielles furent établies dans le discours du groupe blanc, elles furent largement acceptées dans tous les segments de la société. Les principaux mouvements politiques du côté non-blanc furent organisés selon les lignes de ce même système classificatoire. Les indigènes avaient été englobés dans un État unique pour la première fois avec l'établissement de l'Union sud-africaine en 1910. Tandis que l'Union était en train d'être négociée par les groupes politiques blancs, une Convention indigène sudafricaine se tint à Bloemfontein pour protester contre l'exclusion des nonBlancs du droit de vote. En 1912, le Congrès national indigène sud-africain fut créé, et en 1925 il prit le nom de Congrès national africain (African National Congress, ANC). En 1954 fut établie une alliance des partis du Congrès, qui regroupa le Congrès national africain et le Congrès national indien (plus ancien, fondé par Gandhi); à cette occasion apparurent deux groupes nouvellement constitués et numériquement insignifiants, le Congrès des gens de couleur (Coloured Peoples' Congress) et le Congrès des démocrates, dirigé par des Blancs, qui était une organisation de façade du Parti communiste.

Cette division raciale enracinée fut mise en question par un mouvement qui vit le jour à l'université dans les années vingt. Celui-ci affirmait que l'Afrique du Sud était une société unique. Sans doute cette société était-elle traversée par de dangereuses et radicales divisions internes, mais ces divisions provenaient de la structure même de cette société unique. De plus, les différents secteurs de cette société étaient de plus en plus intégrés politiquement et économiquement. La personne qui se fit le meilleur avocat de la thèse de la single society fut l'historien William Miller MacMillan ${ }^{20}$. Selon lui, les promoteurs les plus insidieux de la politique ségrégationniste étaient les ethnologues. Leur stratégie était de ne s'intéresser qu'à des populations tribales, en apparence conservatrices, qui vivaient dans les réserves indigènes. Rejetant l'ethnographie traditionnelle comme obsolète, et se plaignant de ce qu'elle détournait l'attention des problèmes actuels de

19. VAN Warmelo, 1935 , p. 7.

20. Sur William Miller MacMillan, voir Saunders, 1988, p. 47-75; MacMillan, 1975. 
la vie sud-africaine, MacMillan soulignait que dès 1915, «à peine la moitié des indigènes de l'Union semblait avoir une maison, hormis sur des terres possédées [...] par des Européens ${ }^{21} \gg$. Les réserves étaient dans tous les cas grossièrement insuffisantes, même pour les populations qui restaient, et les institutions traditionnelles des Africains ruraux avaient été anéanties.

MacMillan déclara la guerre à tous les anthropologues sans discrimination, mais en réalité les anthropologues sociaux des universités de Cape Town et du Witwatersrand se rangèrent en général à son analyse. Radcliffe-Brown, le premier professeur d'anthropologie sociale d'Afrique du Sud, reconnaissait que l'organisation sociale traditionnelle avait été radicalement ébranlée, et il insistait sur le fait que même l'organisation d'une tribu du Transkaï ne pouvait être comprise que dans le contexte d'une économie et d'une organisation de l'État à l'échelle nationale. D'accord avec les tenants de la thèse d'une société unique selon lesquels cette société connaissait des transformations radicales, les anthropologues «sociaux» tournèrent leur attention vers l'érosion des identités culturelles locales, la croissance de la migration de main d'œuvre, et l'imposition généralisée d'une division binaire entre les Blancs et les Noirs. Le principal ethnographe de l'école anglaise, Isaac Schapera, étudia les changements religieux, économiques et sociaux parmi les Kgatla dans le protectorat du Bechuanaland (actuel Botswana), resituant leurs institutions dans le contexte d'une société coloniale et dans celui plus vaste de l'économie du sud de l'Afrique. Une perspective identique sous-tendait l'ethnographie classique d'une population du Transkaï, intitulée de façon éloquente Reaction to conquest. Effects of contact with Europeans on the Pondo of South Africa (Réactions face à la conquête. Effets du contact avec les Européens sur le Pondo d'Afrique du Sud) publiée en 1936 par Monica Hunter (plus connue sous son nom de femme mariée, Wilson).

En 1934, Schapera dirigea un livre intitulé Western civilization and the natives of South Africa. Studies in culture contact (La Civilisation occidentale et les indigènes d'Afrique du Sud. Études de contact de cultures). Ce livre s'ouvrait sur un chapitre intitulé "La culture bantoue traditionnelle » mais s'attachait ensuite à la description du changement culturel, de la pénurie des terres, de la migration rurale, du christianisme et de l'urbanisation, avec peu de références aux variations culturelles locales ou aux forces traditionnelles. En 1937, Schapera résuma la situation dans le sud de l'Afrique en ces termes :

« Des Bantous en général on peut dire qu'ils ont été à présent attirés dans l'orbite de la civilisation occidentale de façon permanente. Ils ne prolongent

21. MacMillan, 1929, p. 312. 
pas, et probablement ne prolongeront pas cette civilisation dans ses manifestations typiquement européennes. Il est plus vraisemblable que dans certaines directions au moins ils développeront leurs propres variantes locales. Mais ces variantes se feront dans le cadre d'une civilisation sud-africaine commune, partagée par les Noirs et les Blancs, et présentant certaines particularités fondées directement sur leur juxtaposition même. Déjà une telle civilisation est en train de se développer, une civilisation dans laquelle les Européens occupent pour l'instant la position d'une aristocratie privilégiée et fière de sa race, tandis que les indigènes, bien qu'étant indispensables à l'économie, sont cantonnés dans un statut servile duquel peu parviendront à émerger avec succès [...] Mais malgré tout cela, les Bantous participent toujours davantage à la vie culturelle commune de l'Afrique du Sud $[\ldots]^{22}$. »

La thèse de la société unique amena les ethnographes à considérer les communautés rurales dans la perspective de leurs rapports avec l'État, et encouragea des études politiques, économiques et sociales. Toutefois, il y avait bien sûr une alternative habituelle, prédominante dans la plupart des centres métropolitains, qui consistait à étudier les traditions culturelles et les aires culturelles. L'ouvrage The Bantu-Speaking Tribes of South Africa (Les Tribus de langue bantoue en Afrique du Sud), dirigé par Schapera et publié en 1937, ne traitait que des populations de langue bantoue à l'intérieur des frontières de l'Afrique du Sud et des trois protectorats britanniques $^{23}$; il ne tenait pas compte des tribus Nguni et Sotho dans le Zimbabwe et en Zambie, ni des Tsonga au Mozambique; mais l'ouvrage semble avoir été conçu comme une enquête dans une aire culturelle. Il est vrai que des efforts systématiques ont été fournis pour décrire précisément les uniformités de la région bantoue du sud au lieu d'enregistrer les moindres différences tribales locales, ce qui constituait une nouveauté par rapport aux études plus classiques d'aire culturelle ${ }^{24}$. Néanmoins, il faut voir dans cet ouvrage une contribution à une tradition d'études d'aires culturelles plus conventionnelle, et cela représentait, du moins en partie, une alternative aux projets inspirés par la thèse de la société unique. Il est significatif qu'initialement, Schapera et le maître à penser de l'apartheid, Eiselen, devaient codiriger l'ouvrage. Finalement, Eiselen se retira pour prendre un poste dans l'administration indigène.

22. SCHAPERA, 1937a, p. 386-387.

23. Le Swaziland, le Basutoland et le protectorat du Bechuanaland devinrent des colonies britanniques dans les années 1880 . Les frontières coloniales traversaient des unités historiques établies, et aussi bien culturellement que linguistiquement ces pays ont toujours fait partie de l'Afrique du Sud. Ils ont toujours été dépendants de l'Afrique du Sud, économiquement et politiquement. Tout le monde s'attendait à ce qu'ils soient intégrés à l'Union, et il y eut des tentatives régulières pour les inclure jusqu'à ce que l'indépendance leur ait été accordée au milieu des années soixante (Swaziland, Lesotho et Botswana).

24. Une génération plus tard, W. David Hammond-Tooke retint un tel modèle pour l'édition d'une version révisée de l'ouvrage. Voir HAMmond-Tooke, 1974. J'ai publié une critique de cette approche dans un article de revue, voir KuPER, 1976. 
Dans les centres métropolitains de la discipline, la tradition des aires culturelles continuait de prédominer, ou du moins était encore respectable. Même au sein de l'anthropologie sociale britannique, les monographies de Bronislaw Malinowski sur les Trobriand, ou le travail de Charles Seligman et d'Edward Evans-Pritchard au Soudan étaient pour une large part des études anhistoriques de modes de vie supposés traditionnels. Après la Seconde Guerre mondiale, l'étude des changements sociaux fut souvent décrite comme ressortissant du domaine de la sociologie, ce qui sousentendait qu'elle relevait d'une autre discipline, moins prestigieuse ${ }^{25}$. Quand Hilda Kuper fit son compte rendu des Swazi, elle le divisa en deux : une longue première partie consacrée au système traditionnel, et une seconde partie qui traitait des changements de la période coloniale, suite à un système de domination raciale. L'Institut africain international (IAI) décida de ne publier que la première partie (An African aristocracy, 1947), laissant aux presses de l'université de Witwatersrand le soin de publier la seconde (The Uniform of colour, également en 1947).

Il y eut cependant une contre-attaque de la part des anthropologues sudafricains plus radicaux, rassemblés notamment autour de Max Gluckman. Celle-ci contribua à faire entrer la critique sud-africaine des études culturelles au cœur de l'anthropologie sociale britannique ${ }^{26}$. Ancien étudiant de Hoernlé et homme de gauche convaincu, Gluckman publia quelques-unes des analyses les plus innovatrices sur la société rurale en adoptant la perspective de la société unique, en démontrant que même au cœur du Zululand, région réputée pour son conservatisme culturel, le changement culturel et des interactions sociales complexes entre les Blancs et les Noirs modelaient la vie quotidienne ${ }^{27}$.

La prémisse fondamentale de la thèse de la société unique était que les identités tribales ou ethniques s'étioleraient à mesure que les institutions

25. Edward Evans-Pritchard, contre Bronislaw Malinowski, rejetait alors les études de changement social comme étant une branche de l' «anthropologie appliquée », qui comme tout domaine appliqué était d'un point de vue universitaire moins sérieux et intéressant que la forme pure de la discipline. Ce rejet peut s'interpréter comme lié à des considérations de prestige de la discipline dans l'univers d'Oxbridge. Voir L'Estoile, 1997, p. 363-364.

26. KUPER, 2000, chap. vI.

27. Voir, plus particulièrement, GlucKman, 1940. Comme en 1939, le gouvernement sudafricain lui interdit de poursuivre ses recherches sur le terrain au Zululand, il accepta un poste à l'Institut Rhodes-Livingstone en Rhodésie du Nord pendant la Seconde Guerre mondiale. Après la guerre, ses collègues et lui-même furent les premiers à faire l'étude de la politique urbaine et des migrations de mains-d'œuvre en Afrique centrale. En 1949, il fut nommé à l'université de Manchester, à la chaire d'anthropologie sociale nouvellement créée, et il introduisit alors dans l'anthropologie sociale britannique ce qui était pour l'essentiel la théorie de la société unique. 
communes dans les domaines économique, politique et social évolueraient. Dans le même temps, il y aurait une convergence au sein d'une civilisation commune. Le camp opposé était celui des ségrégationnistes, identifié largement au camp nationaliste afrikaner. Pour eux, l'intégration et l'assimilation ethnique n'étaient absolument pas envisageables, car elles représentaient une menace pour l'identité nationale du peuple afrikaner comme pour l'intégrité de la race blanche. Le projet de l'apartheid avait pour but de renforcer les divisions, considérées comme des divisions naturelles, au sein de la population sud-africaine : entre les Afrikaners et les Anglais; entre les Noirs et les Blancs; entre les Asiatiques, les Coloureds et les Bantous à l'intérieur de la population non européenne; et, enfin, au sein de la catégorie des Bantous, entre tribus ou nations.

On fit appel à l'expérience des anthropologues (de langue afrikaans et nationalistes) pour définir à l'intérieur de la catégorie bantoue les sousgroupes appropriés. Van Warmelo, l'anthropologue officiel du gouvernement, fut laissé de côté, ce qui n'était pas étonnant vu son scepticisme visà-vis de la pertinence qu'on attribuait à l'époque aux identités traditionnelles. On tenta de promouvoir une théorie spéciale, qu'on appela la théorie de l'ethnos. Variante de la théorie romantique traditionnelle du peuple (Volk), son hypothèse centrale était que chaque groupe ethnique authentique avait une culture distincte, sans laquelle ses membres se sentiraient perdus et misérables. Une prémisse secondaire était que les membres d'un tel groupe préféraient faire des mariages endogames, de sorte que l'ethnos tendait à devenir un groupe biologique ${ }^{28}$.

Mais en dépit d'une théorie très générale sur l'importance des identités de base, ces ethnologues officiels se trouvaient devant des difficultés insolubles quand il leur fallut identifier les communautés qui partageaient ces identités. Les chefferies africaines du XIX ${ }^{\mathrm{e}}$ siècle avaient été écrasées par les armées boers et britanniques, et quoi qu'il en soit, dans certaines régions, on ne trouvait pas d'unités de taille suffisamment grande pour former la base de divisions administratives modernes. Ce n'était là que des problèmes courants quand on connaît l'Indirect Rule ailleurs en Afrique, mais ils se manifestaient avec encore plus d'acuité ici en raison des changements imposés par un siècle de domination blanche et de société en pleine industrialisation, car ces changements avaient été bien plus radicaux qu'ailleurs sur le continent. Finalement, les Bantous furent officiellement décrits comme une famille de même langue, et divisés en dix unités nationales selon des critères vaguement géographiques et linguistiques. Au sein de

28. L'approche théorique de cette école de l'ethnos (ethnos school) est résumée et discutée in Hammond-Tooke, 1997, chap. vi. 
chaque section, une lignée de chefs suffisamment conservatrice fut désignée et établie en tant que famille dominante ${ }^{29}$.

Les adversaires de l'apartheid ne perdirent pas leur temps à critiquer ces catégories. Aussi bien les critiques libéraux que les radicaux insistaient sur le fait que les divisions inventées ou renforcées par le système de l'apartheid étaient des fictions dangereuses et artificielles, imposées à un système économique et politique de plus en plus intégré (quoique traversé en profondeur par des conflits), au sein duquel de nombreuses valeurs étaient partagées. Le point de vue de la société unique devint l'orthodoxie pour la génération suivante dans les universités de langue anglaise en Afrique du Sud, et à mesure que le système de l'apartheid devenait plus répressif, une nouvelle vague de recherches universitaires reformula en termes marxistes le modèle de la société unique. La piste fut ouverte par les historiens, qui introduisirent une version modifiée du modèle marxiste classique en trois phases $^{30}$. Traditionnellement, les peuples africains avaient vécu dans des petites chefferies fondées sur la descendance et organisées en lignages. Puis, à la fin du XVII ${ }^{\mathrm{e}}$ ou au début du XVIII ${ }^{\mathrm{e}}$ siècle, se produisit une transition générale vers un système d'État quasi féodal. Pour finir, toutes ces unités politiques indépendantes furent conquises par les Blancs et absorbées par l'État capitaliste, et leurs peuples furent réduits à un statut uniforme de paysannerie sans terre et de travailleurs itinérants.

La perspective marxiste qui se répandit dans les universités de langue anglaise ne laissait pas de place à la tradition, à l'ethnicité ou à la culture ; ou plutôt, celles-ci n'apparaissaient que comme des mystifications au service d'une politique qui voulait diviser pour mieux régner. Les nouvelles études ethnographiques menées à bien par des anthropologues dans ces universités avaient pour but de démontrer les effets de l'apartheid. Les objets ethnographiques privilégiés étaient les églises dans les banlieues noires, les foyers de travailleurs migrants et les associations où ceux-ci se retrouvaient, les rituels et les chansons associées avec les migrations des travailleurs, l'impact des migrations sur le groupe domestique rural, et les peuples déplacés installés en tel ou tel lieu en fonction de programmes de repeuplement ou privés de leur droit d'occupation dans les exploitations agricoles des Blancs. Tout cela se déroulait avec, en arrière-plan, une idéologie qui mettait l'accent sur les différences culturelles, non seulement entre les Blancs et les Noirs mais aussi au sein de la population noire, ainsi qu'une politique qui consistait à instituer des gouvernements tribaux pseudo-traditionnels, tandis que l'État s'efforçait, brutalement mais finalement sans succès, d'inverser les flux de population dans les zones urbaines.

29. Hammond-Tooke, 1997, chap. v et vi.

30. SAUNDERS, 1988, p. 165-185. 
La thèse de la société unique renforça la tendance des anthropologues sud-africains de langue anglaise à limiter leur curiosité ethnographique aux frontières de l'État. Cette focalisation fut accentuée par le boycott culturel international des années soixante-dix et quatre-vingt. Les chercheurs sudafricains se refermèrent alors sur eux-mêmes. Peu de comparaisons furent faites avec les pays du Nord, même avec des pays comme le Botswana et le Zimbabwe. Toutefois, le modèle n'encourageait pas à faire des comparaisons au sein de l'Afrique du Sud elle-même. Il était admis que la société unique était divisée verticalement, en classes ou en castes raciales, mais pas horizontalement, en groupes ethniques. Toute prise en considération des différences culturelles était suspecte, car elle pouvait être mise au service des intérêts de l'apartheid. Tous les opprimés étaient dans le même bateau. Les identités ethniques étaient une création, à l'initiative des Blancs ${ }^{31}$.

\section{V}

Les ethnographies sud-africaines classiques, publiées dans les années trente et quarante, portaient sur des populations rurales de langue bantoue. Au cours de cette période, une seule étude de cette nature fut consacrée à un groupe hottentot ou khoï, les Namas. Dans les années soixante, les anthropologues de l'université de Cape Town commencèrent à faire des études ethnographiques sur les communautés coloureds ou hottentotes ${ }^{32}$ au Cap et en Namibie, mais la désignation des populations étudiées était devenue un sujet des plus sensibles.

Légalement, les peuples non blancs parlant l'afrikaans du Cap occidental étaient membres d'une caste spécifique dans le système de l'apartheid. On les appelait Coloureds (gens de couleur). Les intellectuels préféraient l'autodésignation ironique «soi-disant gens de couleur» (so-called Coloureds), mais les raisons avancées pour quelque catégorisation que ce soit donnaient matière à d'âpres disputes. Parmi ceux qui parlaient le nama, la langue hottentote la plus répandue, la plupart parlait aussi couramment l'afrikaans, et les origines hottentotes, de même que le fait d'avoir des ancêtres esclaves, étaient passés sous silence par tous ceux qui étaient concernés. Par ailleurs, il existait des liens culturels évidents avec les Afrikaners. Ils avaient en commun la langue et la religion (ainsi que les noms de famille), mais étaient séparés de ces derniers socialement et politiquement. Dans les années 1960, quelques libéraux Afrikaner demandèrent l'inclusion des Coloureds dans la nation afrikaner, en arguant que l'identité culturelle primait sur la race; mais la stratégie favorite, à la fois parmi les

31. VAIL, 1989.

32. Carstens, 1966. 
activistes politiques et les chercheurs en sciences sociales, était plutôt de les décrire comme étant des Sud-Africains noirs, en mettant en avant le fait que leur conscience et leurs conditions de vie étaient modelées par l'apartheid à l'instar de l'existence du reste du prolétariat privé de droit de vote.

La trajectoire suivie par les études des Boschimans fut très différente. Cela était dû au fait que ce domaine ne relevait pas du champ de l'anthropologie sud-africaine tel qu'il avait été défini. La recherche se concentra sur le Botswana, et s'étendit même jusqu'en Namibie, et en conséquence ne souffrit pas du boycott universitaire. À la suite de l'étude pionnière sur les !Kung menée par Lorna Marshall dans les années cinquante ${ }^{33}$, une nouvelle vague d'ethnographes étrangers se mit à travailler sur le Botswana. Les anthropologues sociaux basés en Afrique du Sud ne manifestèrent pas d'intérêt pour le regain des études sur les Boschimans, qui leur semblaient éloignées de leurs préoccupations. Les axes de la recherche étaient définis selon les directives très différentes de l'anthropologie d'inspiration principalement américaine.

Le grand débat théorique qui secoua les études boschimanes se concentrait sur les !Kung, et notamment sur la définition à donner de leur statut historique et évolutif. La célèbre analyse de Richard Lee fut effectuée dans la tradition de l'évolutionnisme américain. Il fit une description à tendance écologique de l'adaptation des chasseurs-cueilleurs à un environnement semi-désertique, et il présenta ses découvertes comme une contribution à la reconstruction de la transition vers le paléolithique supérieur en Afrique. Les !Kung en vinrent à servir d'exemples ethnographiques d'une étape universelle de l'évolution humaine ${ }^{34}$. Cette vision des choses fut mise en question par une école révisionniste qui estimait que tous les Boschimans avaient été soumis depuis des siècles par des peuples pasteurs, noirs et blancs, auxquels ils avaient été incorporés. Il était absurde de les considérer comme les derniers représentants africains d'un mode de vie antique de chasseurs-cueilleurs. Au contraire, ils étaient la classe inférieure d'un système social unique hiérarchisé ${ }^{35}$.

Il y avait aussi un débat autour de l'identité ethnique de ces peuples, et de leur désignation propre. Selon un procédé familier, des termes coloniaux gênants furent remplacés par une nomenclature scientifique fondée sur une terminologie vernaculaire. En 1930, Schapera avait donné son imprimatur au terme Khoïsan, utilisé pour désigner l'ensemble de la catégorie générale Hottentot-Boschiman. Monica Wilson fut la première anthropologue sudafricaine de la génération suivante à remplacer les termes Boschimans et Hottentots respectivement par San et Khoï, bien que San soit en réalité un

33. Ses essais sur les!Kung ont été rassemblés. Voir Marshall, 1976.

34. LeE, 1979; LeE et De Vore, 1968.

35. WilmsEN, 1989. 
mot péjoratif khoï (hottentot) pour un chasseur. La distinction entre Boschimans et Hottentots fut elle-même mise en question dans les années quatre-vingt. Les classifications linguistiques ne recoupaient pas la division entre les chasseurs et les éleveurs de bétail, et des preuves historiques montraient que certaines populations étaient passées de la chasse à l'élevage avant de revenir à la chasse ${ }^{36}$. Au Botswana, les appellations ethniques ou linguistiques furent contestées par les responsables de projets de développement, qui renommèrent les Boschimans (San) par le terme tswana (péjoratif) de Sarwa, qui fut alors redéfini par les activistes politiques comme signifiant peuples isolés, caractérisés par leur pauvreté et leur faiblesse politique.

Bien que des influences politiques locales soient aussi pertinentes, à partir des années soixante les études boschimanes se développèrent principalement dans le cadre d'un discours anthropologique cosmopolite. On pourrait peut-être voir certains points communs entre les révisionnistes des études boschimanes et les théoriciens de la société unique en Afrique du Sud, car les uns comme les autres insistaient sur les processus d'incorporation et de domination. Mais il est intéressant de constater que les deux groupes ne soulignaient pas la même chose. Pour certains chercheurs sud-africains, il semblait évident que les forces structurantes étaient exercées par l'État ou par le système capitaliste minier centralisé et hautement corporatiste, luimême étroitement associé à l'État. Pour les chercheurs, souvent liés à des institutions américaines, qui travaillaient au Botswana et en Namibie, l'État n'avait aucun rôle. Le pouvoir d'influencer les communautés locales était au contraire attribué aux forces globales indéterminées de l'impérialisme et du capitalisme qui jouent un rôle si central dans la démonologie moderne de la gauche américaine.

Du point de vue de la construction de l'objet ethnographique, les courants principaux de l'anthropologie sud-africaine et de l'ethnographie boschimane ont convergé vers une question commune : la relation entre un groupe ethnique d'une part, et un État ou un Empire de l'autre. Ce vieux problème était posé d'une façon nouvelle. Aussi bien les tenants de la société unique en Afrique du Sud que les révisionnistes du Botswana s'accordaient à dire que le groupe ethnique avait été inventé par une puissance supérieure, poursuivant des motifs méprisables : l'État, le discours impérial, ou le système capitaliste. La tâche des chercheurs était d'abord de déconstruire cette fiction, et ensuite de constituer de nouveaux objets de recherche. Ceux-ci étaient à nouveau définis par rapport à l'État, mais étaient identifiés comme étant les produits réels de l'exploitation économique et politique. En Afrique du Sud, les victimes de l'apartheid

36. BaRnARD, 1992, chap. II. 
devinrent les objets de l'anthropologie. Au Botswana, l'attention fut transférée des Boschimans ou San vers l'underclass, ce quart-monde à la merci du tiers.

\section{VI}

La transition vers un système politique démocratique fonctionna comme une gigantesque expérience sociale qui mit à l'épreuve les théories des chercheurs. La vieille théorie de l'apartheid prédisait que les Africains exprimeraient toujours leur loyauté primordiale aux identités tribales. Toutefois, il devint rapidement clair que l'ANC avait un immense soutien parmi les Sud-Africains noirs presque partout dans le pays. De manière très significative, quelques-uns des vieux partis des Bantoustans avaient aussi conservé un certain soutien dans les régions où l'on parlait le zoulou; mais même là, l'électorat était nettement divisé en deux : un secteur rural au cœur du KwaZulu, qui restait loyal au mouvement nationaliste zoulou Inkhata, et un secteur urbain dans le Natal, généralement chrétien, qui votait pour l'ANC ${ }^{37}$.

Il y avait aussi d'autres bizarreries locales. Ainsi, l'électorat des Coloureds et des Indiens était divisé, des ensembles non négligeables de ces groupes votant pour des partis blancs ${ }^{38}$. Toutefois, la grande séparation politique qui apparut à l'échelle nationale correspondait pour l'essentiel aux divisions raciales qui avaient été imposées depuis le milieu du XIX ${ }^{\mathrm{e}}$ siècle. On pourrait avancer que l'élément décisif était la classe, et non la race, car un siècle de discrimination raciale avait produit une correspondance étroite entre la race, l'éducation, le logement, le type d'emploi et le revenu. Il n'est pas facile de séparer ces facteurs, mais il reste qu'une immense majorité des électeurs africains soutint l'ANC, et la plupart des Blancs votèrent pour les partis historiquement blancs ${ }^{39}$.

Rien de tout cela n'a surpris les anthropologues de langue anglaise. Toutefois, ils commencent à reconnaître qu'ils ont des difficultés à conceptualiser les processus culturels et sociaux qui émergent dans la nouvelle Afrique du Sud. Dans une introduction à une récente collection d'essais d'anthropologues sud-africains de langue anglaise, Patrick McAllister remarque que leur discipline est en train «d'émerger du lieu où elle s'était elle-même coincée lors des jours sombres de l'apartheid», pendant lesquels elle avait été caractérisée par «sa préoccupation pour des analyses

37. Cette division entre une population rurale conservatrice et une population des banlieues et des villes progressistes avait été un thème central de l'ethnographie de langue anglaise.

38. Lors des deux premières élections libres en Afrique du Sud, en 1994 et 1999, une majorité de Coloureds vota pour le Parti nationaliste afrikaner.

39. LODGE, 1999, voir particulièrement chap. III. 
d'économie politique»- «économie politique» étant l'euphémisme, emprunté aux Américains, pour désigner le marxisme. Ses collègues et luimême ont dû alors reconnaître que «la culture doit être réintroduite dans les réponses que nous donnons pour expliquer la pratique sociale ${ }^{40} \gg$. Dialoguant (non sans étonnement) avec son ancien Soi marxiste, un autre anthropologue sud-africain, Andrew Spiegel, remarque que les ethnographes actifs dans la campagne doivent admettre que

« quelque chose [du passé précolonial] a survécu (ou semble avoir survécu), a persisté ou s'est poursuivi, et qu'on ne peut supposer que l'incorporation dans un environnement industriel a abouti à un retournement complet et un remplacement de ce qui existait ou s'était produit auparavant ${ }^{41} »$.

Ce sont là quelques brindilles lancées pour trouver la direction du vent, indiquant de manière encore schématique les orientations suivies par un travail ethnographique qui s'efforce de prendre davantage en compte les conceptions locales du monde. Il existe cependant d'autres possibilités. Un certain nombre d'anthropologues, anglais et afrikaners, se sont réfugiés dans un relativisme postmoderne. D'autres encore ont abandonné les problèmes abstraits de la théorie en faveur de la recherche appliquée, très en demande aujourd'hui. Les anciens projets de recherche étaient motivés par une opposition à l'apartheid, au capitalisme, au colonialisme. Le défi est à présent de servir l'État, plutôt que de s'y opposer. Mais une recherche responsable sur le plan social ne saurait suffire. Il sera bientôt nécessaire de répondre au défi intellectuel lancé par l'ANC. Le nouvel État est en train de développer ses propres vues de l'Afrique du Sud. La discrimination positive est appliquée légalement. Le président Mbeki encourage des discussions sur une « renaissance africaine », et il commence à accueillir favorablement les idées afrocentriques. Peut-être les anthropologues vont-ils devoir aborder de nouveaux débats sur des thèmes familiers, en discutant une fois de plus les questions les plus fondamentales, comme celles du caractère de la société unique et de la nature des unités qui constituent la société sud-africaine.

\section{Adam KuPER ${ }^{42}$ \\ (avril 2000).}

\footnotetext{
40. McAllister, 1997, p. 2-3.

41. SPIEGEL, 1997 , p. 10.

42. Je suis très reconnaissant à Benoît de L'Estoile pour ses suggestions stimulantes sur une version antérieure de cet article, et à Isaac Schapera pour sa relecture. Traduction : Yann THOLONIAT.
} 


\section{LISTE DES RÉFÉRENCES}

Barnard (Alan), 1992, Hunters and herders of Southern Africa. A comparative ethnography of the Khoisan peoples, Cambridge, Cambridge University Press.

Carstens (Peter), 1966, The Social Structure of a Cape Coloured Reserve, Cape Town, Oxford University Press.

GLUCKMAN (Max), 1940, «Analysis of a social situation in modern Zululand », Bantu Studies, vol. XII, p. 147-174.

Gordon (Robert), 1990, «Early social anthropology in South Africa », African Studies, vol. XLIX, 1, p. 15-48.

Gordon (Robert) et SpIEGEL (Andrew D.), 1993, « South African anthropology revisited », Annual Review of Anthropology, vol. XXII, p. 83-105.

Hamilton (Carolyn), 1998, Terrific majesty, Cambridge, MA, Harvard University Press.

Hammond-Tooke (W. David), éd., 1974, The Bantu-Speaking Peoples of Southern Africa, Londres, Routledge and Kegan Paul.

Hammond-Tooke (W. D.), 1997, Imperfect interpreters. South Africa's anthropologists, 1920-1990, Johannesburg, Witwatersrand University Press.

HARRIES (Peter), 1989, «Exclusion, classification and internal colonialism. The emergence of ethnicity among the Tsonga-speakers of South Africa », in VAIL, 1989.

HunTER (Monica), 1936, Reaction to conquest. Effects of contact with Europeans on the Pondo of South Africa, Londres, Oxford University Press.

Junod (Henri A.), 1912, The Life of a South African tribe, Neuchatel, Imprimerie Attinger, 2 vol.

KIERNAN (Jim), 1997, « David in the path of Goliath. South African anthropology in the shadow of apartheid», in MCAllister, 1997.

KUPER (Adam), 1976, «Culture area or political system?», Africa, vol. XLVI, 3, p. 291-294.

KUPER (A.), 1999, Among the anthropologists, Londres, Athlone Press.

Kuper (A.), 2000, L'Anthropologie britannique au Xx ${ }^{e}$ siècle, $1^{\mathrm{re}}$ éd. 1973, ici trad. de l'anglais par Gérald Gaillard, Paris, Karthala.

KuPER (Hilda), 1947a, An African aristocracy. Rank among the Swazi, Londres, Oxford University Press for the International African Institute.

KuPER (H.), 1947b, The Uniform of colour. A study of White-Black relationships in Swaziland, Johannesburg, Witwatersrand University Press.

LeE (Richard B.), 1979, The !Kung San. Men, women, and work in a foraging society, Cambridge, Cambridge University Press.

Lee (Richard B.) et De Vore (Irven), éd., 1968, Man the hunter, Chicago, Aldine.

L'Estoile (Benoît de), 1997, "The "natural preserve of anthropologists". Social anthropology, scientific planning and development », Social Science Information, vol. XXXVI, 2, June, p. 343-376.

Lodge (Tom), 1999, Consolidating democracy, Johannesburg, Witwatersrand University Press.

MacMillan (William Miller), 1929, Bantu, Boer, and Briton. The making of the South African native problem, Londres, Faber and Gwyer.

MacMillan (W. M.), 1975, My South African years, Cape Town, David Philip. 
Marshall (Lorna), 1976, The !Kung of Nyae Nyae, Cambridge, MA, Harvard University Press.

McAllister (Patrick), éd., 1997, Culture and the commonplace, Johannesburg, Witwatersrand University Press.

Ramphele (Mamphela), 1995, A life, Cape Town, David Philip.

Ross (Robert), 1993, Beyond the pale. Essays on the history of colonial South Africa, Hanover, Wesleyan University Press.

SAUNDERS (Christopher), 1988, The Making of the South African past. Major historians on race and class, Cape Town, David Philip.

Schapera (Isaac), 1930, The Khoisan Peoples of South Africa. Bushmen and Hottentots, Londres, George Routledge \& Sons.

Schapera (I.), éd., 1934, Western civilization and the natives of South Africa. Studies in culture contact, Londres, George Routledge \& Sons.

Schapera (I.), 1937a, « Cultural changes in tribal life », in Schapera, $1937 \mathrm{~b}$.

Schapera (I.), éd., 1937b, The Bantu-Speaking Tribes of South Africa. An ethnographical survey, Londres, George Routledge \& Sons.

Spiegel (Andrew D.), 1997, «Continuities, culture and the commonplace. Searching for a new ethnographic approach in South Africa », in McAllister, 1997.

Vail (Leroy), éd., 1989, The Creation of tribalism in Southern Africa, Londres, James Currey.

VAn Rensburg (Fanie) et VAn der WaAl (Kees), 1999, « Continuity and change in South African cultural anthropology (Volkekunde). Issues of essentialism and complexity », South African Journal of Ethnology, vol. XXII, 2, p. 45-48.

VAN Warmelo (Nicholas J.), 1935, A preliminary survey of the Bantu tribes of South Africa, Pretoria, The Government Printer.

VAwDA (Shahid), 1995, « The other anthropology. A response to Gordon and Spiegel's review of Southern African anthropology », African Studies, vol. LIV, 1, p. 128-131.

WeST (Martin), 1988, Social anthropology in a divided society, Cape Town, University of Cape Town (Inaugural Lecture Series).

Wilmsen (Edwin N.), 1989, Land filled with flies. A political economy of the Kalahari, Chicago, University of Chicago Press. 